\title{
Life after prostate cancer: A systematic literature review and thematic synthesis of the post-treatment experiences of Black African and Black Caribbean men
}

\author{
O. Bamidele MSC, BSC, PhD Student ${ }^{1}$ (D) ～H. McGarvey PhD, BSC (HONS), PGCTHE, RNT, \\ RN, Lecturer in Nursing ${ }^{2}$ (D) | B.M. Lagan PhD, MSC, BSC, RNT, RGN, RM, Lecturer in Nursing \\ and Public Health ${ }^{1}$ (D) | N. Ali PhD, MSC, BSC (HONS), Senior Research Fellow in Public \\ Health $^{3}$ | F. Chinegwundoh MBE MBBS, MS, MML (Med Law), FRCS(Eng.), FRCS(Ed), \\ FRCS(Urol), FEBU, Consultant Urological Surgeon ${ }^{4,5}$ | K. Parahoo PhD, BA, RMN, \\ Professor in Nursing and Health Research ${ }^{6}$ | E. McCaughan PhD, BSC, PGDHE, PGCUT, DIP, \\ ONCOLOGY NURSING, RGN, Professor in Cancer Care ${ }^{6}$
}

\footnotetext{
${ }^{1}$ Institute of Nursing and Health Research, Ulster University, Jordanstown, UK

${ }^{2}$ School of Nursing, Ulster University, Londonderry, UK

${ }^{3}$ Institute of Health Research, University of Bedfordshire, Luton, UK

${ }^{4}$ Barts Health NHS Trust, London, UK

${ }^{5}$ City, University of London, School of Health Sciences, London, UK

${ }^{6}$ Institute of Nursing and Health

Research, Ulster University, Coleraine, UK

Correspondence

Olufikayo Omowumi Bamidele, MSC, BSC, PhD Student, Institute of Nursing and Health Research, Ulster University, Jordanstown, UK Email: Bamidele-O@email.ulster.ac.uk

Funding information

This review was undertaken as part of a PhD studentship to OB supported by an Ulster University Vice-Chancellor's Research Scholarship
}

Evidence shows that there are significant ethnic variations in prostate cancer prevalence and outcomes. Specifically, Black African and Black Caribbean men may encounter different post-treatment experiences than Caucasian men due to their disproportionately higher risk of being diagnosed with advanced prostate cancer. But to date, no review of these experiences has been undertaken. This review synthesised findings from existing literature on the post-treatment experiences of Black African and Black Caribbean men with prostate cancer and identified pertinent issues which may be useful to inform practice and future research. Seven databases were systematically searched using developed search terms. Four qualitative studies were identified and critically appraised. Findings are summarised under four main themes: symptom experience, healthcare experience, marital and social relationships and coping strategies. Cultural definitions of masculinity influenced the meanings men gave to their post-treatment experiences. While men's experiences of healthcare varied, the provision of professional support to address their post-treatment distress was lacking. Men derived most support from wives, peers and church communities. A culturally sensitive approach which recognises diversity among Black African and Black Caribbean populations and treats individuals within their religious and socio-cultural contexts could potentially improve men's post-treatment experiences. Areas for further research were also identified.

KEYWORDS

Black African, Black Caribbean, experience, men, post-treatment, prostate cancer 


\section{1 | INTRODUCTION}

In addition to reducing the prevalence of cancer, the National Health Service (NHS) England's cancer strategy also aims to speed up cancer diagnosis leading to better treatments, improved healthcare and long-term quality of life for cancer survivors (NHS England, 2016). For prostate cancer, evidence shows that there are significant ethnic variations in its prevalence and outcomes (Marmot et al., 2010; Prostate Cancer UK (PCUK), 2014a). Specifically, Black African (BA) and Black Caribbean (BC) men have two to three times higher risk of being diagnosed with advanced prostate cancer than Caucasian men (Ber cblomo et al., 2008; Bray, Ren, Masuyer, \& Ferlay, 2013; Willian 2 14; Rebbeck et al., 2013). Although older men are more at risk of prostate cancer, among $\mathrm{BA}$ and $\mathrm{BC}$ men, the risk is also increasing for younger men (Ben-Shlomo et al., 2008; PCUK, 2014b).

A number of reasons for ethnic variations in prostate cancer prevalence and outcomes have been presented in the literature. These include: genetic-environmental interactions (Ben-Shlomo et al., 2008; Chornokur, Dalton, Borysova, \& Kumar, 2011; Odedina et al., 2011; Rebbeck et al., 2013), knowledge of cancer and cancer symptoms (Pedersen, Armes, \& Ream, 2012; Rajbabu et al., 2007), cultural barriers including the role of masculinity (being the breadwinner, being 'tough', demonstrating sexual prowess and virility), fatalistic beliefs, superstitious beliefs, loss of sexuality, and stigma (Mulugeta, 2014; Pedersen et al., 2012). Available evidence also highlights, that Black and Minority Ethnic (BME) groups report poor experiences of healthcare due to a lack of cultural sensitivity in healthcare delivery (Das-Munshi, Leavey, Stansfeld, \& Prince, 2012; Pinder, Ferguson, \& Moller, 2016). As a consequence, a combination of factors contributes to delays in seeking help for prostate cancer (Pedersen et al., 2012), resulting in late diagnosis, poorer post-treatment outcomes and higher mortality rates among BA and BC populations (Jones \& Chinegwundoh, 2014; Rebbeck et al., 2013).

With improved access to early diagnosis and treatment, survival rates from prostate cancer have increased and many men now live up to at least five to 10 years post-diagnosis in the absence of co-morbidities (Cancer Research UK, 2014). As a result of these advances in treatment and improved survival rates, men who survive prostate cancer are living longer with post-treatment complications such as sexual/erectile problems, incontinence issues, pain, 'menopause-like' feelings and fatigue (Gavin et al., 2015). Such treatment side effects have been shown to affect men's ability to perform their daily activities and roles including: work (Grunfeld, Drudge-Coates, Rixon, Eaton, \& Cooper, 2013; Roth, Weinberger, $\&$ Nelson, 2008), marital role as the breadwinner and sexual partner (Coomarsingh, 2012; Gerressu, 2016; Phillips, 2005), therefore impacting on their psychosocial wellbeing and quality of life (Gavin et al., 2015; McCaughan, McKenna, McSorley, \& Parahoo, 2015; McCaughan et al., 2013).

The National Institute of Health and Care Excellence (NICE) recommend the provision of appropriate support care to help men deal with the side effects of prostate cancer treatment (NICE, 2015). To deliver such post-treatment support care, there is need for cultural sensitivity, taking into account the cultural interpretation of prostate cancer among BA and BC populations, and how this may influence their experiences and coping with the disease. There is however, a dearth of evidence showing the post-treatment experiences of BA and BC men and how they cope with prostate cancer, including its impact on their masculinity. Previous reviews have reported the experiences of a Caucasian sample (e.g. King et al., 2015) or BME group as a whole (Rivas et al., 2016), but have not specifically explored the role of ethnicity in the post-treatment experiences of BA and $\mathrm{BC}$ men with prostate cancer. Those that have had a BA and $\mathrm{BC}$ focus, have looked at knowledge and perceptions of prostate screening (Pedersen et al., 2012) or their support needs (Cremin, 2015). Hence, this review aims to address this knowledge gap by synthesising findings from existing literature on the post-treatment experiences of $\mathrm{BA}$ and $\mathrm{BC}$ men with prostate cancer, and identify pertinent issues that need to be addressed to ensure improved satisfaction with care, better post-treatment experiences and improved quality of care for this target group. In addition, the review aims to explore areas for future research.

\section{2 | METHODS}

As recommended by Aveyard (2014), a systematic approach was adopted in identifying relevant studies to address the review aim.

\subsection{Search strategy}

Existing literature was systematically searched to identify articles relating to the post-treatment experiences of prostate cancer among BA and BC men. The search was conducted between November 2015 and June 2016 across seven bibliographic databases namely: CINAHL plus, Medline Ovid, Psych Info, Cochrane Central, Embase, ProQuest and Scopus. Search terms were developed using the following key words: prostate, cancer, prostatic neoplasm, experience, coping, outcome, treatment outcome, post-treatment, Black African, Black Caribbean, African, Caribbean, African-Caribbean, Afro-Caribbean, Black. Truncation (*) and quotation mark (") were used where appropriate to capture all variants of keywords whilst Boolean operators 'OR' and 'AND' were also used to broaden and limit search results as appropriate.

Choice of database to search and development of search terms were made in consultation with an experienced subject librarian. An author search was conducted on Scopus to identify similar and related articles from authors in the research area and journal alerts were set up for research updates. The grey literature was also searched for unpublished empirical research (dissertations, conference proceedings, research reports). Finally, the reference lists of selected papers were hand-searched whilst main authors were also contacted via email for possible updates to their papers and their knowledge of any other relevant studies. 


\section{2 | Inclusion and exclusion criteria}

A set of inclusion and exclusion criteria (Table 1) were set to ensure a focused, credible and significant review within a feasible time frame (Centre for Reviews and Dissemination 2009). Initial searches showed that there was a dearth of research evidence relating to post-treatment experiences of BA and BC men with prostate cancer. Therefore, the authors considered it reasonable not to exclude articles based on publication date or study setting. Hence, empirical studies which addressed the post-treatment experiences of prostate cancer among BA and BC men, and published in English were included in the review. Studies including different types of cancers were included if they had separate data on prostate cancer. Publications which involved both Black and Caucasian participants, but did not report their respective views separately, were excluded. Studies related to African American men were also excluded because they are the indigenous Blacks in America and their sociocultural experiences may be different from that of immigrant $B A$ and $B C$ men (Agyemang, Bhopal, \& Bruijnzeels, 2005; Odedina et al., 2011).

\section{3 | Screening}

A Prisma diagram of the study illustrating the process used to select articles relevant to be included in the review is presented in Appendix A. The initial search yielded 932 publications from which 702 duplicates were removed. The title and abstracts of the remaining 230 publications were then read and a further 93 were considered irrelevant and removed. The remaining 137 papers were assessed for inclusion by two authors (OB and $\mathrm{EM})$. Two other authors (BL and HM) provided a second opinion where needed. Four research studies were considered eligible for this review. None of the four publications were identified from grey literature.

\section{4 | Data extraction and critical appraisal}

Data were managed using an extraction sheet created on Microsoft Excel (2016). The four selected papers were further reviewed by five authors (OB, EM, HM, BML and $\mathrm{KP}$ ) and differences in opinion around data extraction and appraisal were resolved via discussion. The methodological quality and trustworthiness of selected studies

TABLE 1 Inclusion and exclusion criteria

\begin{tabular}{|c|c|}
\hline Inclusion criteria & Exclusion criteria \\
\hline $\begin{array}{l}\text { Studies which provided } \\
\text { empirical data on the } \\
\text { post-treatment experiences of } \\
\text { BA and BC men with prostate } \\
\text { cancer }\end{array}$ & $\begin{array}{l}\text { Studies on the post-treatment } \\
\text { experiences of men with prostate } \\
\text { cancer where the views of BA } \\
\text { and BC participants were not } \\
\text { reported separately }\end{array}$ \\
\hline \multirow[t]{4}{*}{ Published in English language } & $\begin{array}{l}\text { Studies involving African American } \\
\text { men }\end{array}$ \\
\hline & $\begin{array}{l}\text { Studies which focused on prostate } \\
\text { screening }\end{array}$ \\
\hline & $\begin{array}{l}\text { Epidemiological and intervention } \\
\text { studies }\end{array}$ \\
\hline & Anecdotal evidence \\
\hline
\end{tabular}

were appraised using the Critical Appraisal Skills Programme (CASP, 2013) tool for qualitative research (Appendix B).

\subsection{Identification and synthesis of themes}

All four selected studies were qualitative, therefore, we carried out a thematic synthesis based on the approach recommended by Thomas and Harden (2008). Descriptive themes were developed by mapping participants' views and experiences in each of the papers in relation to the review aim. Discussions among members of the research team (OB, EM, HM and KP) facilitated a mutual agreement on the descriptive themes which emerged across the papers reviewed. This was then followed by comparison and interpretation of findings across the different studies to generate new analytical constructs (Thomas $\&$ Harden, 2008)

\section{3 | RESULTS}

\section{1 | Overview of papers}

The four qualitative studies were published between 2005 and 2013 (Anderson, Marshall-Lucette, \& Webb, 2013; Bache, Bhui, Dein, \& Korszun, 2012; Gray, Fergus, \& Fitch, 2005; Nanton \& Dale, 2011). All four studies had used individual interviews to explore the posttreatment experiences of $\mathrm{BA}$ and $\mathrm{BC}$ men with prostate cancer, in relation to their respective study aims. Table 2 presents, in a chronological order, detailed characteristics of the four selected studies. Each of the selected studies had undergone peer-review prior to being published in their respective journals. A critical appraisal of each study demonstrated good methodological quality (Appendix B). Therefore, all four studies were included in this review. With regards to their usefulness, the studies by Gray et al. (2005) and Nanton and Dale (2011) provided more in-depth and richer data to meet the aim of this review compared with the studies by Bache et al. (2012) and Anderson et al. (2013). Nevertheless, the latter two studies were also included as they had BA participants in their studies and the limited evidence they presented was still considered useful to provide a glimpse into the experiences after prostate cancer for this grossly under-researched population.

Three out of the four studies were conducted in the UK (Anderson et al., 2013; Bache et al., 2012; Nanton \& Dale, 2011) whilst the fourth study was conducted in Canada (Gray et al., 2005). The demographic and clinical details of the participants in the studies were not fully and consistently reported. Hence, what emerged was a patchy profile with missing data. A total of 27 participants (22 BC, 4 BA men and 1 unknown) aged between $50-83$ years old were interviewed across the four studies. Many participants were married ( $n=12$, marital status of others were not stated) and in blue collar jobs (manual/wage-earning [ $n=13]$ ). The treatment received by most participants included: active surveillance $(n=2)$, radical prostatectomy $(n=12)$, radiotherapy $(n=2)$, radiotherapy with hormone therapy $(n=5)$, prostatectomy with radiotherapy and hormone therapy $(n=1)$. The length of time since treatment ranged from ongoing treatment to 15 years post-diagnosis. Demographics of 


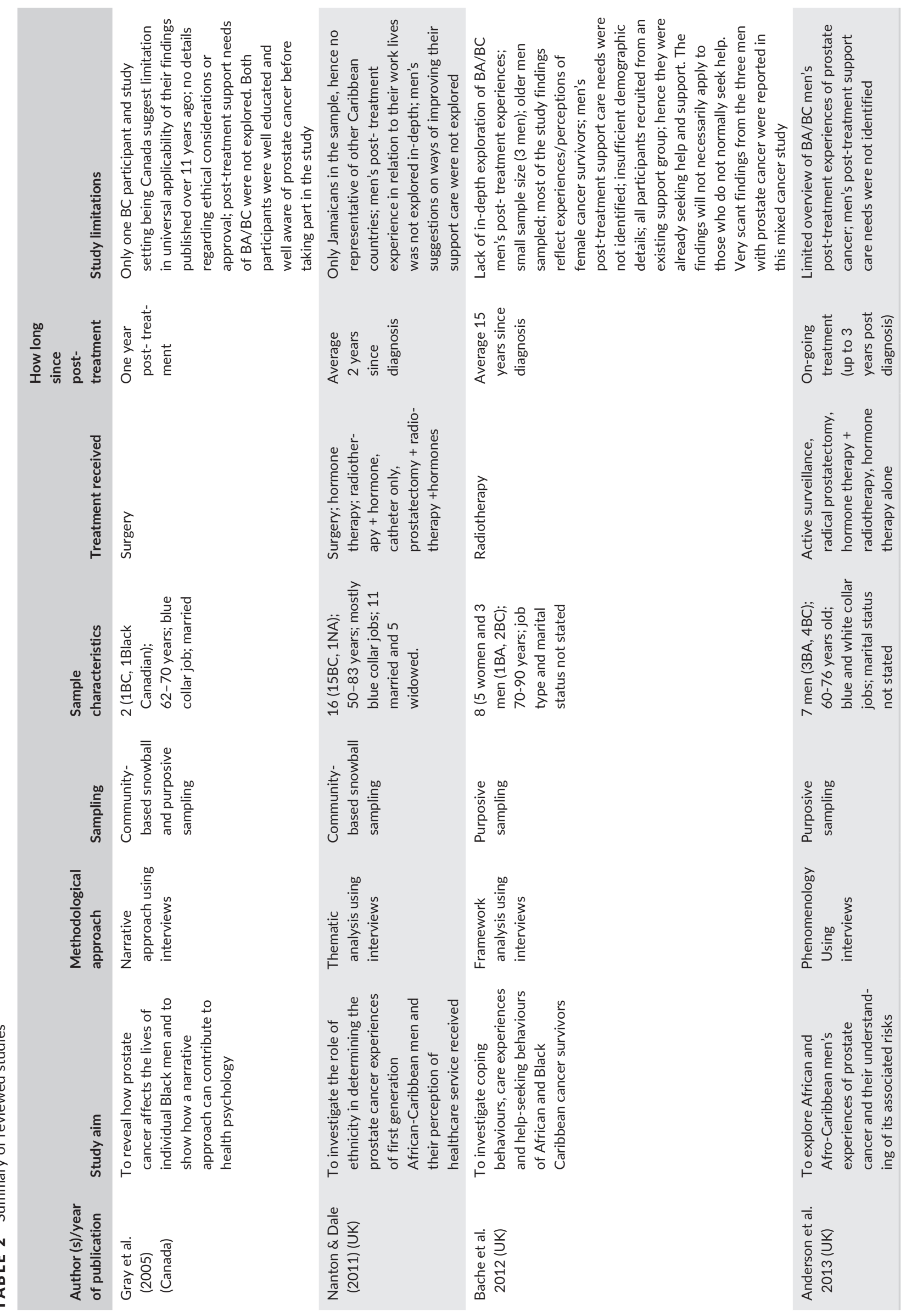


the sample for each of the four studies is presented in Table 2. Thirteen descriptive themes emerged across the reviewed papers and were categorised into four broader themes namely: symptom experience, healthcare experience, marital and social relationships and coping strategies.

\section{2 | Symptom experience}

Whilst variations existed in the type and severity of treatment side effects experienced, sexual dysfunction following treatment emerged as a generally shared post-treatment complication which affected men across all the studies. Although some men also reported distressing experience of urine incontinence (Nanton \& Dale, 2011), others reported that they were spared from incontinence issues after their prostate cancer treatment (Gray et al., 2005).

\subsection{1 | Dealing with treatment side effects}

A recurring and prominent theme across three of the four studies related to sexual problems following prostate cancer and how these participants perceived masculinity and their marital relationships (Anderson et al., 2013; Gray et al., 2005; Nanton \& Dale, 2011). Findings from the review showed that sexual potency was a significant determinant of men's personal feelings of their masculine identity and this in turn influenced their help-seeking for prostate cancer (Anderson et al., 2013; Gray et al., 2005; Nanton \& Dale, 2011). Men were as concerned over partial and uncertain erections (Gray et al., 2005) as they were about complete sexual dysfunction posttreatment (Anderson et al., 2013; Nanton \& Dale, 2011):

'there are things that come into your mind now that prevent you from enjoying sex' (Gray et al., 2005; participant quote)

However, some men expressed the fear of losing their wives due to their inability to provide sexual satisfaction following their illness:

'Also when the chemo [therapy] was really having its effect on me, I was really afraid that she [wife] would leave me' (Anderson et al., 2013; participant quote)

In dealing with their sexual problems, some men were prescribed medications by their doctors (Nanton \& Dale, 2011) whilst others reported struggle in dealing with such sexual difficulties (Gray et al., 2005). In spite of their advanced years in age and co-morbid conditions with diabetes which appeared to have compounded reduced sexual functioning in some participants, men expressed deep feelings of disappointment at their sexual loss post-treatment. As one recollected from a conversation with a doctor:

'you can't do that as when you were young age man, but me tell him I can accept that but it shouldn't have all gone' (Nanton \& Dale, 2011; participant quote)
Daily management of post-treatment incontinence was also a major concern to most participants in Nanton and Dale's (2011) study as this had caused significant restrictions on many aspects of their lives. Beyond physical limitations to men's daily lives, incontinence problems affected men psychosocially with some reporting feelings of stress, embarrassment and inconvenience, due to delayed and limited supply of incontinence aids by healthcare professionals.

\subsection{Healthcare experience}

Disparities were observed across the four studies in men's individual perceptions of their healthcare experiences. Some men reported more positive experiences (Bache et al., 2012; Gray et al., 2005) than others (Nanton \& Dale, 2011). BA and BC men's perceptions of their healthcare experience were reflected across four subthemes namely: decision-making for treatment choice, treatment side effects, experience of healthcare services and communication with healthcare professionals.

\subsection{1 | Decision-making}

Men's involvement in the decision-making process for their treatment choice and managing post-treatment side effects varied across and within the studies reviewed. Some men were more engaged than others in the decision-making process with their healthcare professionals (Bache et al., 2012; Gray et al., 2005; Nanton \& Dale, 2011) whereas others relied solely on their doctor's prescription, and seldom asked questions regarding treatment choice or how to manage side effects (Bache et al., 2012; Nanton \& Dale, 2011). Increased knowledge and awareness through personal research and consultation with known peer survivors also contributed to some men's engagement with their doctors to decide treatment options (Gray et al., 2005; Nanton \& Dale, 2011). Other men admitted little prostate cancer awareness prior to their diagnosis (Nanton \& Dale, 2011). Although generally men expressed confidence in their doctor's competence, some felt pressure to comply with certain prescribed treatments as these were viewed to be a natural course of the prostate cancer trajectory:

'It was something like compulsory that they...you have to have it because I first started to rebel against this injection. The doctor even said to me, he said 'forget how many hundred pounds it costs for one of these injections' and 'I am fortunate to be given the type of treatment.' (Nanton \& Dale, 2011; participant quote)

'I'd have said they are the boss, you know they said radiotherapy, check it out, could be best for you. I just get on with it. I don't force.' (Bache et al., 2012; participant quote) 


\subsection{2 | Experience of healthcare service}

Diverse reactions in men's perceptions of the healthcare service were identified from the review. Positive reports of the healthcare service were mostly associated with: successful treatment outcomes facilitated by doctors' technical skills (Gray et al., 2005), doctors' communication skills (Bache et al., 2012), availability of radical treatment procedures (Nanton \& Dale, 2011) and the presence of a Black nurse post-surgery, which was perceived as an indication of culturally sensitive caring (Gray et al., 2005). Although there were no reports of obvious racial discrimination in any of the studies, some participants felt that some healthcare professionals resorted to stereotyping and described Black men as being "secretive and reluctant to seek treatment" (Gray et al., 2005). Men's concerns regarding side effects of hormone therapy were also perceived by some healthcare professionals as a masculinity issue (Nanton \& Dale, 2011):

'And I explained to the doctor...I said, 'I don't want to have this injection' because it's a thing that bloats you up see and I don't want to do that ...it gives you breasts and I said 'No' and he's telling me about being like macho man and I said 'No, I do not want to be a macho man, I just want to be me.' (Nanton \& Dale, 2011; participant quote)

Several participants expressed negative experiences of the healthcare service which they attributed to hasty and unsympathetic attitude devoid of respect for personal dignity from some healthcare professionals. Such negative experiences were further compounded by lack of adequate information and support to deal with post-treatment side effects especially with regards to incontinence (Nanton \& Dale, 2011). Most men did not report being offered any psychosocial support posttreatment but the few who were signposted to support agencies complained of complex referral procedures and inadequate services which resulted in feelings of disappointment with such services (Nanton \& Dale, 2011):

'(I went to see) the welfare rights and she was explaining to me that I could get help for certain things, but... like, 'why don't you try the Macmillan...?' but by the time you're phoning here and phoning there, I said 'I can't worry about it' (Nanton \& Dale, 2011; participant quote)

\subsubsection{Communication with healthcare professionals}

With the exception of men in the study by Bache et al. (2012) who reported receiving full information prior to treatment, this review established from the other three studies (Anderson et al., 2013; Gray et al., 2005; Nanton \& Dale, 2011) that there was limited communication between men and healthcare professionals especially in relation to post-treatment side effects or how to deal with such. This further impacted on the men's perception of their healthcare experience.
However, men's perceptions of such limited conversations differed across the reviewed studies. Some men reported that their doctor's candid prescription of surgery without mentioning potential side effects boosted their confidence and enhanced their coping with the disease (Gray et al., 2005). Others noted that their doctors were not sensitive when communicating their prostate cancer diagnosis and that the little information they received made them less prepared for the treatment side effects they experienced (Nanton \& Dale, 2011). When men had reservations about certain aspects of their care, some did not communicate their uncertainties to a healthcare professional. Men also noted cultural barriers in discussing sexual matters with healthcare professionals:

'my opinion is this: I think most African and Caribbean men find it a taboo to talk about anything apart from normal illness, anything about sex life, they think it's a bit taboo to discuss it with their doctors or GPs, especially if they have a female GP.' (Anderson et al., 2013; participant quote)

In spite of the varied reactions to existing limited doctor-patient communication, men generally expressed a desire for more information through rapport-building between doctor and patient to help them (patients) cope with prostate cancer and its treatment side effects (Gray et al., 2005; Nanton \& Dale, 2011):

'You're not going to sit there and tell me about my prostate and that's all we talk about. No, we talk about other things too.' (Gray et al., 2005; participant quote)

\subsection{Marital and social relationships}

Sexual difficulties after prostate cancer treatment and the potential impact on their intimate marital relationships were reported by men in all the four studies. They also admitted communication barriers in discussing such sexual concerns with their wives. Incontinence issues were also seen to restrict men's daily personal and social lives.

\subsection{1 | Discussing sexual concerns with wives}

Although many participants in most of the studies reviewed (Anderson et al., 2013; Gray et al., 2005; Nanton \& Dale, 2011) experienced sexual problems after their prostate cancer treatment, there was no indication of communication between couples regarding such sexual issues. A participant in one of the studies admitted that difficulty in discussing sexual concerns with his wife was due to 'lack of camaraderie' existent in the Caribbean culture (Gray et al., 2005). The same participant also stressed that discussing sexual issues with wives and initiating sex could pose an additional burden for men at this stage of their prostate cancer journey. Men noted that their inability to meet with cultural expectations to be a leader in their marriage(s) could potentially lead to dysfunction in their marital relationships posttreatment for prostate cancer. 
There were suggestions that health professionals could serve as intermediaries in facilitating marital communication by engaging wives in active discussions on their new roles to initiate sexual activity as a way of helping to deal with their husband's sexual difficulties after prostate cancer treatment. One participant went further in advocating how this could be achieved:

'he should have called her into the office and said, these are your new responsibilities, stick to them and it would help, and these are the reasons why it would help, and be beneficial in the end.' (Gray et al., 2005; participant quote).

\subsubsection{Work life}

Whilst the impact of prostate cancer treatment on men's working lives was not explored in most of the reviewed studies, one participant's narratives in Gray et al. (2005) showed a positive experience as his prostate cancer advanced his 'prominence' both within and outside his workplace. Such prominence was reflected in this participant's increased request to be involved in the development of novel health promotion activities at his workplace, which further contributed to his perception of positive post-treatment experience.

\subsection{Coping strategies}

There were variations across these four studies regarding how men coped with their prostate cancer. Different internal and external factors enhanced better coping in some men than others.

\subsection{1 | Resilience}

Participants in the four studies (Anderson et al., 2013; Bache et al., 2012; Gray et al., 2005; Nanton \& Dale, 2011), generally showed a resilient attitude towards their prostate cancer. Such resilience was more observed among the younger men as many of them were reported to have received their diagnosis calmly (Nanton \& Dale, 2011) in spite of their fatalistic beliefs about prostate cancer and initial anxiety over potential loss of sexual function (Anderson et al., 2013; Nanton \& Dale, 2011). In some cases, men's resilience was motivated by their desire to carry on with life (Bache et al., 2012) and continue living a happy family life post-prostate cancer (Anderson et al., 2013). Some men noted that their personal willpower enhanced their successful surgery and coping post-surgery (Gray et al., 2005). Others who perceived going through the diagnostic and treatment processes as imperative, maintained this resilient attitude along their post-treatment journey (Anderson et al., 2013) and resolved to manage their prostate cancer on a day-to-day basis (Nanton \& Dale, 2011):

'there is certain amount of reluctance or whatever it is... to have to go, but my own personal situation; I felt that if something was wrong, painful and ugly as it all might have been, that had to go through with it' (Anderson et al., 2013; participant quote)

'so it has given me extra resolve not to brood about the fact that I have prostate cancer.' (Bache et al., 2012; participant quote)

\subsection{2 | Religious faith}

A key finding across the studies was the adaptation of religious faith as a coping strategy. Many men described a personal conviction in the ability of God to help them cope with their prostate cancer (Bache et al., 2012; Nanton \& Dale, 2011). Some men reported that prayers from others also contributed to their successful surgery (Gray et al., 2005). They described how their religious beliefs not only empowered them to think positively about their diagnosis, but also gave them the strength to accept and manage resulting post-treatment issues (Bache et al., 2012):

'Oh it has strengthened my mind because it has given me the necessary will power to accept what has come to me and also to accept the consequences, Yes' (Bache et al., 2012; participant quote)

'you see as I always call on the Lord and all of these things I said 'Lord Jesus help me to go through this thing' and then I go through it and I came home' (Nanton \& Dale, 2011; participant quote)

\subsection{3 | Traditional remedies}

Although not a recurring theme across other studies, most men in Nanton and Dale's (2011) study portrayed a staunch belief in the use of traditional remedies and have continued to use them to complement their medical treatment for their prostate cancer. Even though this was predominant among the older participants in the study, younger men who were less familiar with such traditional remedies also used dietary supplements in addition to their medical treatment:

'...they say pumpkin seeds are good for you, so I started to buy some' (Nanton \& Dale, 2011; participant quote)

\subsubsection{Social support}

The review showed that $\mathrm{BA}$ and $\mathrm{BC}$ men with prostate cancer had very limited sources of professional support. Most of their posttreatment support had been provided by their wives, immediate families, other peer survivors and the church. In some instances, and especially among those without access to such support, there were feelings of helplessness: 
'I don't know who to go to for help here' (Nanton \&

Dale, 2011; participant quote)

Referral to support organisations was only mentioned in the Nanton \& Dale's study (2011) and the few men involved expressed disappointment at the services received which they described as unsuitable to meet their post-treatment needs. Even where study participants were recruited through a support group (Bache et al., 2012), their support experience and perceptions of services provided by such organisation were not explored.

\subsubsection{Wife and other family support}

The majority of married men described their wives as very supportive along their prostate cancer journey (Gray et al., 2005; Nanton \& Dale, 2011). Men in Anderson et al.'s (2013) study also expressed fear of being abandoned by their wives because of how prostate cancer complications affected their sexual abilities but most participants in the three other studies (Anderson et al., 2013; Gray et al., 2005; Nanton \& Dale, 2011) did not report such concerns. Wives steered the men to seek help for their cancer symptoms and also provided them with support to live with prostate cancer (Gray et al., 2005; Nanton \& Dale, 2011).

'...She has been extremely supportive' (Gray et al., 2005; participant quote)

Older men and those without wives or family members nearby reported feeling isolated and having poorer coping abilities than those with wives. Men who were carers for their wives and/or for a family member with an illness or disability also reported less able to cope with their prostate cancer than those with no caring responsibilities (Nanton \& Dale, 2011).

\subsection{6 | Peer support from and for others with prostate cancer}

Findings identified that communicating with known peer survivors promoted greater confidence and greatly enhanced the ability of most BA and BC men to cope with their prostate cancer (Anderson et al., 2013; Gray et al., 2005; Nanton \& Dale, 2011). However, there were variations across the studies in how men navigated such peer-support relationships. Whereas some men were direct beneficiaries of peer-support activities in seeking early diagnosis (Anderson et al., 2013), making treatment decisions (Gray et al., 2005; Nanton \& Dale, 2011) and dealing with post-treatment issues (Nanton \& Dale, 2011), others derived personal satisfaction from engaging in prostate cancer buddy activities which further enhanced their personal coping with their disease (Anderson et al., 2013; Gray et al., 2005):

'It's [prostate cancer] killing people in droves. Some have had the operation done, it's done poorly, and they are in pain. Some talk to me because they know l've had it done. I'm willing to talk about it' (Anderson et al., 2013; participant quote)

In instances where their masculinity ideals were constructed around status and social relationship with peers, men noted that their disease experience further reinforced their prostate advocacy activities. This earned them increased recognitions and made them more 'famous' as peer supporters for other men with prostate cancer (Gray et al., 2005).

\subsection{7 | Support from the church}

Findings further showed that the church provided a highly valued source of support for BA and BC men with prostate cancer. Men acknowledged that the spiritual and moral support offered by the church were helpful on their disease journey:

'Well, apart from wife my church stands by me with my illness and my friends. Everybody who knew that you were ill will encourage you and that is the help I get. I didn't get it from any (other) organisations.' (Nanton \& Dale, 2011; participant quote)

\section{4 | DISCUSSION}

This review aimed to synthesise findings from existing literature on the post-treatment experiences of $\mathrm{BA}$ and $\mathrm{BC}$ men with prostate cancer and identify pertinent issues that need to be addressed to ensure improved satisfaction with care, better post-treatment experiences and improved quality of care for this target group. The review also aimed to explore areas for future research. Findings from the review show that the experiences of $\mathrm{BA}$ and $\mathrm{BC}$ men with prostate cancer are similar to that of their Caucasian counterparts (King et al., 2015). There are however some indications within the findings of the four identified studies that there can be complex interactions between culture, age, gender and socio-economic factors. There are indications that culture may play an important part in shaping the experiences of BA and BC men posttreatment for prostate cancer than has been previously reported. We now discuss the findings from the four papers under three main themes: sexual dysfunction as a cultural threat to masculine identity, perception and use of healthcare services, and coping and social support.

\subsection{Sexual dysfunction as a cultural threat to masculine identity}

The threat prostate cancer presents to masculine identities is well documented for Caucasian men (Appleton et al., 2015; Beck, Robinson, \& Carlson, 2009; McCaughan et al., 2015; Roth et al., 2008). Evidence highlights that socio-cultural context plays an important role in the dominant constructions of what it is to be a man (or woman) (Peplau, Veniegas, Taylor, \& DeBro, 1999). BA and BC masculine identities centre on their sexuality which is defined by virility and prowess (Case \& Gordon, 2016). For example, the expectation that men will have 
more than one sexual partner (Coomarsingh, 2012; Gerressu, 2016; Plummer, 2013) and engage in sexual activities at an earlier age than their Caucasian counterparts is well documented (Gerressu, 2016). For some of the participants in the studies reviewed, sexual dysfunction (physical and psychological) was a main concern impacting on them performing their masculine identities. The fear of being abandoned by their wives was also mentioned by some participants (Anderson et al., 2013) in this review. The self and social stigma associated with not being able to perform their masculine sexual roles has been noted as one of the causes of delays in seeking treatment for prostate cancer and post-treatment psychosocial stress among Black men (Kendrick, 2010; Mulugeta, 2014; Pedersen et al., 2012).

Sexual dysfunction in men can therefore 'upset' the balance in male-female relationships because it undermines dominant cultural ideas of BA and BC men's masculinity. In one of the studies in this review (Gray et al., 2005), a participant commented on the 'lack of camaraderie' that he thinks is common in Caribbean marriages. He found it difficult to talk about sexual dysfunction with his wife and wished that the urologist had 'ner into the office' and told her about 'her new responsibilities' ( $p .40$. However, many Caribbean women have been noted to lack the power to determine when and how to have sex (Allen, 1997; Coomarsingh, 2012). If this is the case, relinquishing their customary leadership roles in initiating sexual activity may be hard for these men. Arguably, such cultural stereotypes and beliefs may have changed over time among these ethnic migrants due to acculturation to their host country (Taitt, 2015). Nevertheless, Brown (2014) argues that despite inter-ethnic acculturations, people still uphold a substantial degree of their indigenous cultural beliefs and attitudes which often influence their health behaviours and play a pivotal role in observed ethnic inequalities in health experiences and outcomes.

\section{2 | Perception and use of healthcare services}

Similar to their Caucasian counterparts (King et al., 2015), men in the reviewed studies reported positive and negative experiences with healthcare services. Technical skills of their doctors in carrying out successful treatment procedures, mostly accounted for the men's perceptions of positive healthcare experience (Gray et al., 2005). However, unmet information needs, communication barriers in discussing their sexual difficulties with healthcare professionals and perceptions of cultural stereotypes from healthcare professionals, contributed to negative experiences of healthcare reported by many participants (Anderson et al., 2013; Gray et al., 2005; Nanton \& Dale, 2011). These further resonate with findings from previous studies (Papadopoulos \& Lees, 2004; Viruell-Fuentes, Miranda, \& Abdulrahim, 2012) in which language barrier and cultural stereotypes contributed to perceptions of poor healthcare experiences among ethnic minority populations.

BA and BC men's experiences of stereotyping from healthcare professionals and their (men's) perception of discussing sexual concerns as a 'taboo', highlight the need for cultural sensitivity in patient-healthcare professional communication in relation to prostate cancer. Pedersen et al. (2012) further reported that African-Caribbean men often showed a lack of trust in healthcare professionals and the healthcare system, mainly due to inadequate communication with health professionals. A national survey of the experiences of patients with cancer in the UK showed that Black patients with cancer reported less positive experiences of communication and collaborative care from healthcare professionals, compared with their Caucasian counterparts (Quality Health 2013). However, there were no data relating specifically to prostate cancer in this survey. There is need, therefore, to understand why there is lack of trust in healthcare professionals (Pedersen et al., 2012) and the reasons for the low satisfaction the Black population has with healthcare services in the UK.

\subsection{Coping and social support}

Related to findings from studies involving Caucasian populations (Beck et al., 2009; Wootten et al., 2014), this review also showed that most $\mathrm{BA}$ and $\mathrm{BC}$ men were stoical in the way they coped with their condition and that wives played vital roles in providing daily support and motivating their men to seek help with their prostate cancer (Gray et al., 2005; Nanton \& Dale, 2011). Narratives of poorer posttreatment coping among men without wives (Nanton \& Dale, 2011) echo findings from Kazer et al. (2011) in which participants without partners in their study identified feelings of loneliness and greater psychological distress than those with partners.

However, despite BA and BC men's reports of psychological distress associated with sexual dysfunction and urinary incontinence posttreatment (Anderson et al., 2013; Nanton \& Dale, 2011), there appears to be a lack of desirable professional support to help the men deal with such distress. Previous reviews on psychosocial interventions for prostate cancer survivors (Chambers, Pinnock, Lepore, Hughes, \& O'Connell, 2011; McCaughan et al., 2013; Northouse et al., 2007) also suggest that Black men are being marginalised in this area. Hagen, GrantKalischuk, and Sanders (2007) noted that men's experiences of prostate cancer treatment are largely influenced by how much professional support and information they receive along their disease journey. Even where some men had been signposted to support services, men described such services as inappropriate and unsuitable to meet their needs (Nanton \& Dale, 2011). Yet, reasons for such assessment or men's suggestions on areas for improvements were not reported in the study.

Close family members and friends, in particular other peers with prostate cancer, formed part of the support network for men in this review. The church emerged as a major source of social support for the men, most of whom also mentioned religious faith as a coping strategy for their prostate cancer (Bache et al., 2012; Gray et al., 2005; Nanton $\&$ Dale, 2011). The emergence of religious faith as a coping strategy in this review emphasises the prominent role of religion in the health experiences of ethnic minority populations (Koenig, 2012). Among the many ethnic groups in the UK, Black people have the highest rate of regular church attendance than other BME populations (TEARFUND, 2007). This is further reflected in the increasing number of majority Black churches in the UK (Akhazemea, 2015). Black populations report consistently higher church attendance (48\%) compared to their Caucasian counterparts (15\%) in the UK (TEARFUND, 2007). Findings 
from a recent systematic review on 'prostate cancer and supportive care' (King et al., 2015) also revealed that out of twenty studies (which included Caucasians and other ethnic groups), only two studies identified church support; both studies had involved BC (Nanton \& Dale, 2011) and African American participants (Rivers et al., 2012) respectively. This is further evidence of the interaction between culture and religion among most BA and BC populations (Akhazemea, 2015; Baruth et al., 2013). The church supports BA and BC populations by providing an enabling social network of individuals who share similar cultural values (Akhazemea, 2015). Collaborating with church leaders could potentially promote the development of culturally appropriate, yet spiritually acceptable interventions that will address the individual needs of BA and BC men with prostate cancer (Baruth et al., 2013).

\subsection{Study limitations and directions for future research}

This review provides useful evidence on the post-treatment experiences of BA and BC men, indicating the centrality of culture in these experiences and highlighting key implications for healthcare practice. However, review findings may not be generalisable to the wider BA and $\mathrm{BC}$ population in the UK due to a number of limitations. The review comprised of only four studies. Although we had used an extensive search strategy, with the expert guidance of an experienced librarian, and also set up journal alerts to enable us to keep up to date with new research during the review period, including only studies published in English Language may have resulted in potentially relevant studies being missed in this review. Most of the narratives in the four studies included in this review, rews of BC men (most of whom originated from Jamaica). It is essential to further explore the views of a more heterogeneous sample of BA and other BC men in order to gain more insight into their unique needs and how these can be best addressed. Lack of details regarding ethnicity in many participants' quotes in the studies made it difficult to differentiate the views of their BA participants from those of the BC men. This further makes the task of separating the intricate characteristics of these similar but different ethnic populations, more complex.

Many participants in the reviewed studies appeared to be well educated and positively inclined towards research participation and therefore may not be representative of the less educated, hard to research and less inclined to seek help BAs and BCs. Future research should devise more robust recruitment strategies which also incorporate snowballing through peer contact and religious leaders to access these hard-to-reach populations and make their voices heard.

There are some indications that partners of men with prostate cancer often undergo similar, or sometimes greater psychosocial distress (such as anxiety and depression) than the men themselves (Nelson \& Kenowitz, 2013). Evidence related to Caucasian and African American populations show differing patterns in what contributes to men and their partners' psychosocial distress after prostate cancer treatment. Whereas men express greater concern regarding their sexual potency, partners report being more adversely affected by their men's experiences of urinary incontinence (Couper et al., 2006) and fear of losing their men to prostate cancer (Rivers et al., 2011). The focus of this review was men themselves and none of the four studies made reference to the post-treatment experiences of partners of BA and BC men with prostate cancer nor identified their specific support needs. Research into partners' experience and needs relating to their men's prostate cancer is sorely needed.

Post-treatment incontinence usually poses the most challenging aspect of most men's prostate cancer experience in relation to their ability to return to paid work (work forms a crucial part of men's masculine identity) (Grunfeld et al., 2013). However, the impact of prostate cancer on the work lives of BA and BC men was not explored in any of the studies in this review. Noting that socio-economic advancement is a major motivating factor for BA and BC men's migration from Africa and the Caribbean (Brown, 2014; Nanton \& Dale, 2011), there is need for future studies to explore the impact of prostate cancer treatment on the working lives of these under-researched ethnic men.

\section{5 | IMPLICATIONS FOR PRACTICE}

Findings from this review suggest the need for healthcare professionals to consider cultural issues without resorting to stereotypes in healthcare delivery for BA and BC men with prostate cancer. Undeniably, culture is a complex yet important determinant of health which requires in-depth understanding in order to address the post-treatment needs of minority ethnic men with prostate cancer. There is need to be cognisant of each individual's disease experience and unique information/ communication needs and ensure that potential post-treatment side effects and how to manage such are clearly communicated to men. Wives/partners should also be included in such discussions and the specific ways in which they can help or be helped along their husbands'/ partners' prostate cancer journey should be identified and addressed.

Healthcare professionals' intervention in facilitating intimate conversations among couples could also potentially help relieve undesirable sex-related burden among $\mathrm{BA}$ and $\mathrm{BC}$ men with prostate cancer. A person-centred approach which recognises the diversity among $B A$ and $\mathrm{BC}$ populations and treats individuals within their religious and sociocultural contexts could potentially promote improved healthcare experiences among prostate cancer survivors within these high-risk populations.

\section{NEW CONTRIBUTION TO KNOWLEDGE}

\section{1 | What we already know}

BA and BC men have a disproportionately higher risk of developing prostate cancer but they are currently under-represented in research related to men's experiences and coping after prostate cancer treatment.

\section{2 | What we do not know}

Very little is known on how culture shapes the meanings BA and BC men attribute to their prostate cancer and how this in turn influences 
their experiences and coping with the disease. Stand-alone evidence from the few individual studies on BA and BC men with prostate cancer do not adequately address this research gap.

\subsection{What our review contributes to existing literature}

This is the first (to the best of our knowledge) systematic literature review which solely focuses on the post-treatment experiences of $\mathrm{BA}$ and $\mathrm{BC}$ men in relation to their cultural context. Our review highlights the need for increased cultural awareness which is devoid of stereotyping, among health professionals caring for men with prostate cancer. This review further provides broader and more in-depth evidence on BA and BC men's post-treatment experiences than in any of the individual studies, amplifying the men's cultural interpretation of prostate cancer and how their experiences differed from those of Caucasian men. The review also identified the church as a potential resource to reach out to $\mathrm{BA}$ and $\mathrm{BC}$ men in order to develop culturally appropriate, yet spiritually acceptable interventions that could improve their experiences after prostate cancer treatment.

\section{7 | CONCLUSIONS}

This review has identified that the post-treatment experiences of BA and $\mathrm{BC}$ men treated for prostate cancer can be different from those of Caucasian men. Sexual dysfunction following treatment, posed a particular threat to participants' identity as males of Caribbean and African descent. This in turn, affected the men's relationships with their partners. There were also varying levels of satisfaction among the $B A$ and $B C$ men in the identified studies regarding health services. Most support for these men was from wives/partners and their church communities. Finally, a number of areas for future research have been suggested. In particular, the experience of partners should be explored.

\section{ACKNOWLEDGEMENT}

The authors would like to thank Mary Rose Holman for her expert guidance with conducting the search for this review.

\section{ORCID}

O. Bamidele iD http://orcid.org/0000-0003-2235-9463

H. McGarvey (iD http://orcid.org/0000-0001-9145-8778

B.M. Lagan (iD http://orcid.org/0000-0001-5536-394X

\section{REFERENCES}

Agyemang, C., Bhopal, R., \& Bruijnzeels, M. (2005). Negro, Black, Black African, African Caribbean, African American or what? Labelling African origin populations in the health arena in the 21st century. Journal of Epidemiology and Community Health, 59, 1014-1018.
Akhazemea, D. (2015). The reshaping of religious and social landscape of Britain: The influence of the black majority churches. Available at: https://www.biblesociety.org.uk/uploads/content/bible_in_transmission/files/2015_spring/BiT_Spring_2015_Akhazemea.pdf (Accessed February 8 2017).

Allen, C. (1997). Researching STD's in the Caribbean. In E. Leo-Rhynie, B. Bailey, \& C. Barrow (Eds.), Gender: A Caribbean multidisciplinary perspective. Kingston, Jamaica: Ian Randle Publishers.

Anderson, B., Marshall-Lucette, S., \& Webb, P. (2013). African and AfroCaribbean men's experiences of prostate cancer. British Journal of Nursing, 22, 1296-1307.

Appleton, L., Wyatt, D., Perkins, E., Parker, C., Crane, J., Jones, A., ... Pagett, M. (2015). The impact of prostate cancer on men's everyday life. European Journal of Cancer Care, 24, 71-84.

Aveyard, H. (2014). Doing a literature review in health and social care: A practical guide (3rd Edition). Maidenhead, England: Open University Press.

Bache, R. A., Bhui, K. S., Dein, S., \& Korszun, A. (2012). African and Black Caribbean origin cancer survivors: A qualitative study of the narratives of causes, coping and care experiences. Ethnicity \& Health, 17, 187-201.

Baruth, M., Wilcox, S., Saunders, R. P., Hooker, S. P., Hussey, J. R., \& Blair, S. N. (2013). Perceived environmental church support and physical activity among black church members. Health Education \& Behavior, 40, 712-720.

Beck, A. M., Robinson, J. W., \& Carlson, L. E. (2009). Sexual intimacy in heterosexual couples after prostate cancer treatment: What we know and what we still need to learn. Urologic Oncology: Seminars and Original Investigations, 27, 137-143.

Ben-Shlomo, Y., Evans, S., Ibrahim, F., Patel, B., Anson, K., Chinegwundoh, F., ... Persad, R. (2008). Prostate cancer: The risk of prostate cancer amongst black men in the United Kingdom: The PROCESS Cohort Study. European Urology, 53, 99-105.

Bray, F., Ren, J., Masuyer, E., \& Ferlay, J. (2013). Global estimates of cancer prevalence for 27 sites in the adult population in 2008. International Journal of Cancer, 132, 1133-1145.

Brown, M. (2014). African and African-Caribbean Londoners' experiences of cancer services: A narrative approach. Doctoral thesis, University of West London.

Cancer Research UK. (2014). Survival statistics for prostate cancer. Available at: http://www.cancerresearchuk.org/about-cancer/type/ prostate-cancer/treatment/statistics-and-outlook-for-prostate-cancer (Accessed July 21 2016).

Case, A.D., \& Gordon, D. M. (2016). Contextualizing the health behavior of Caribbean men. In J. L. Roopnarine \& D. Chadee (Eds.), Caribbean psychology: Indigenous contributions to a global discipline. Washington, DC: American Psychological Association.

Centre for Reviews and Dissemination. (2009). Systematic reviews: CRD's guidance for undertaking reviews in healthcare. Available at: http://www.york. ac.uk/crd/SysRev/!SSL!/WebHelp/SysRev3.htm (Accessed July 18 2016).

Chambers, S. K., Pinnock, C., Lepore, S. J., Hughes, S., \& O'Connell, D. L. (2011). A systematic review of psychosocial interventions for men with prostate cancer and their partners. Patient Education and Counseling, 85, e75-e88.

Chornokur, G., Dalton, K., Borysova, M. E., \& Kumar, N. B. (2011). Disparities at presentation, diagnosis, treatment, and survival in African American men, affected by prostate cancer. The Prostate, 71, 985-997.

Coomarsingh, K. (2012). "Tie the Heifer, Loose the Bull": Gender Inequality in the Caribbean. Available at: http://www.whatispsychology.biz/genderinequality-in-caribbean. (Accessed February 14 2017).

Couper, J., Bloch, S., Love, A., Macvean, M., Duchesne, G. M., \& Kissane, D. (2006). Psychosocial adjustment of female partners of men with prostate cancer: A review of the literature. Psycho-Oncology, 15, 937-953.

Cremin, M. (2015). Meeting the unmet support needs of BME men with prostate cancer. British Journal of Nursing, 24, 20.

Critical Appraisal Skills Programme. (2013). Making sense of evidence. Available at: http://www.casp-uk.net/checklists (Accessed January 23 2016). 
Das-Munshi, J., Leavey, G., Stansfeld, S., \& Prince, M. (2012). Migration, social mobility and common mental disorders: Critical review of the literature and meta-analysis. Ethnicity \& Health, 17, 17-53.

Gavin, A. T., Drummond, F. J., Donnelly, C., O'Leary, E., Sharp, L., \& Kinnear, H. R. (2015). Patient-reported 'ever had' and 'current' long-term physical symptoms after prostate cancer treatments. BJU International, 116 397-406.

Gerressu, M. (2016). Understanding Poor Sexual Health in Black British/ Caribbean Young Men in London: A Qualitative Study of Influences on the Sexual Behaviour of Young Black Men. Doctoral thesis, University College London.

Gray, R. E., Fergus, K. D., \& Fitch, M. I. (2005). Two Black men with prostate cancer: A narrative approach. British Journal of Health Psychology, 10, 71-84.

Grunfeld, E. A., Drudge-Coates, L., Rixon, L., Eaton, E., \& Cooper, A. F. (2013). "The only way I know how to live is to work": A qualitative study of work following treatment for prostate cancer. Health Psychology, 32, 75.

Hagen, B., GrantKalischuk, R., \& Sanders, J. (2007). Disappearing floors and second chances: Men's journeys of prostate cancer. International Journal of Men's Health, 6, 201-223.

Jones, A. L., \& Chinegwundoh, F. (2014). Update on prostate cancer in black men within the UK. Ecancermedicalscience, 8, 455.

Kazer, M. W., Harden, J., Burke, M., Sanda, M. G., Hardy, J., \& Bailey, D. E., \& PROSTQA Study Group. (2011). The experiences of unpartnered men with prostate cancer: A qualitative analysis. Journal of Cancer Survivorship, 5, 132-141.

Kendrick, R. D. (2010). Erectile dysfunction: A barrier to prostate cancer screening among African American men. Doctoral Thesis, Capella University.

King, A., Evans, M., Moore, T., Paterson, C., Sharp, D., Persad, R., \& Huntley, A. (2015). Prostate cancer and supportive care: A systematic review and qualitative synthesis of men's experiences and unmet needs. European Journal of Cancer Care, 24, 618-634.

Koenig, H. G. (2012). Religion, spirituality, and health: The research and clinical implications. ISRN Psychiatry, 2012, 278730. doi: 10.5402/2012/278730

Marmot, M. G., Allen, J., Goldblatt, P., Boyce, T., McNeish, D., Grady, M., \& Geddes, I. (2010). Fair society, healthy lives: The Marmot Review. Strategic review of health inequalities in England post-2010. Available at: file:///C:/Users/Olufikayo/Downloads/fair-society-healthy-lives-executive-summary.pdf (Accessed July 17 2016).

McCaughan, E., McKenna, S., McSorley, O., \& Parahoo, K. (2015). The experience and perceptions of men with prostate cancer and their partners of the CONNECT psychosocial intervention: A qualitative exploration. Journal of Advanced Nursing, 71, 1871-1882.

McCaughan, E., Prue, G., McSorley, O., Northouse, L., Schafenacker, A., \& Parahoo, K. (2013). A randomized controlled trial of a self-management psychosocial intervention for men with prostate cancer and their partners: A study protocol. Journal of Advanced Nursing, 69, 2572-2583.

Mulugeta, B. (2014). The influence of culture on the Views of Black African/ African-Caribbean Men Living in the UK towards Cancer. Doctoral thesis, University of Central Lancashire.

Nanton, V., \& Dale, J. (2011). 'It don't make sense to worry too much': The experience of prostate cancer in African-Caribbean men in the UK. European Journal of Cancer Care, 20, 62-71.

National Institute for Health and Care Excellence. (2015). Prostate Cancer. Available at: http://www.nice.org.uk/guidance/qs91/chapter/Qualitystatement-4-Managing-adverse-effects-of-treatment (Accessed December 18 2015).

Nelson, C. J., \& Kenowitz, J. (2013). Communication and intimacyenhancing interventions for men diagnosed with prostate cancer and their partners. Journal of Sexual Medicine, 10, 127-132.

NHS England. (2016). Achieving world-class cancer outcomes: a strategy for England 2015-2020-one year on 2015-16. Available at: https://www. england.nhs.uk/wp-content/uploads/2016/10/cancer-one-year-on. pdf (Accessed May 17 2017).

Northouse, L. L., Mood, D. W., Schafenacker, A., Montie, J. E., Sandler, H. M., Forman, J. D., ... Kershaw, T. (2007). Randomized clinical trial of a family intervention for prostate cancer patients and their spouses. Cancer, 110, 2809-2818.

Odedina, F. T., Dagne, G., LaRose-Pierre, M., Scrivens, J., Emanuel, F., Adams, A., ... Odedina, O. (2011). Within-group differences between native-born and foreign-born Black men on prostate cancer risk reduction and early detection practices. Journal of Immigrant and Minority Health, 13(6), 996-1004.

Papadopoulos, I., \& Lees, S. (2004). Cancer and communication: Similarities and differences of men with cancer from six different ethnic groups. European Journal of Cancer Care, 13, 154-162.

Pedersen, V. H., Armes, J., \& Ream, E. (2012). Perceptions of prostate cancer in Black African and Black Caribbean men: A systematic review of the literature. Psycho-Oncology, 21, 457-468.

Peplau, L. A., Veniegas, R. C., Taylor, P. C., \& DeBro, S. C. (1999). Sociocultural perspectives on the lives of women and men. In L. A. Peplau, S. C. DeBro, R. C. Veniegas, \& P. C. Taylor (Eds.), Gender, culture and ethnicity (pp. 223-237). Mountain view, CA: Mayfield.

Phillips, S. P. (2005). Defining and measuring gender: A social determinant of health whose time has come. International Journal for Equity in Health, 4, 11.

Pinder, R.J., Ferguson, J., \& Moller, H. (2016). Minority ethnicity patient satisfaction and experience: Results of the National Cancer Patient Experience Survey in England. British Medical Journal Open, 6, e011938.

Plummer, D. C. (2013). Masculinity and risk: How gender constructs drive sexual risks in the Caribbean. Sexuality Research and Social Policy, 10, 165-174.

Prostate Cancer UK. (2014a). Men United v Prostate Cancer: Five Inequalities, five solutions. Available at: http://prostatecanceruk.org/ media/2339836/inequalities-report.pdf (Accessed July 19 2016).

Prostate Cancer UK. (2014b). Remember, remember: Four things all Black men should know about prostate cancer. Available at: http://prostatecanceruk. org/about-us/news-and-views/2014/10/four-things-every-blackman-should-know (Accessed July 19 2016).

Quality Health. (2013). National Cancer Patient Experience Survey 2012-13 National Report. NHS England. Available at: https://www.quality-health. co.uk/resources/surveys/national-cancer-experience-survey/2013national-cancer-patient-experience-survey-reports/301-2013-nationalcancer-patient-experience-survey-programme-national-report/file (Accessed September 25th 2017).

Rajbabu, K., Chandrasekera, S., Zhu, G., Dezylva, S., Grunfeld, E., \& Muir, G. (2007). Racial origin is associated with poor awareness of prostate cancer in UK men, but can be increased by simple information. Prostate Cancer and Prostatic Diseases, 10, 256-260.

Rebbeck, T. R., Devesa, S. S., Chang, B. L., Bunker, C. H., Cheng, I., Cooney, K., ... Zeigler-Johnson, C. M. (2013). Global patterns of prostate cancer incidence, aggressiveness, and mortality in men of african descent. Prostate Cancer, 2013, 560857.

Rivas, C., Matheson, L., Nayoan, J., Glaser, A., Gavin, A., Wright, P., Wagland, R., \& Watson, E. (2016). Ethnicity and the prostate cancer experience: A qualitative metasynthesis. Psycho-Oncology, 25 , 1147-1156.

Rivers, B. M., August, E. M., Gwede, C. K., Hart, A., Donovan, K. A., PowSang, J. M., \& Quinn, G. P. (2011). Psychosocial issues related to sexual functioning among African-American prostate cancer survivors and their spouses. Psycho-Oncology, 20, 106-110.

Rivers, B. M., August, E. M., Quinn, G. P., Gwede, C. K., Pow-Sang, J. M., Green, B. L., \& Jacobsen, P. B. (2012). Understanding the psychosocial issues of African American couples surviving prostate cancer. Journal of Cancer Education, 27, 546-558. 
Roth, A. J., Weinberger, M. I., \& Nelson, C. J. (2008). Prostate cancer: Psychosocial implications and management. Future Oncology (London, England) 4, 561-568.

Taitt, H. E. (2015). Prostate Cancer and Afro-Caribbean Men: Experiences, Perceptions, and Beliefs. Doctoral thesis, Walden University.

TEARFUND. (2007). Churchgoing in the UK. Available at: http://news.bbc. co.uk/1/shared/bsp/hi/pdfs/03_04_07_tearfundchurch.pdf (Accessed February 8 2017).

Thomas, J., \& Harden, A. (2008). Methods for the thematic synthesis of qualitative research in systematic reviews. BMC Medical Research Methodology, 8, 1.

Viruell-Fuentes, E. A., Miranda, P. Y., \& Abdulrahim, S. (2012). More than culture: Structural racism, intersectionality theory, and immigrant health. Social Science \& Medicine, 75, 2099-2106.

Williams, M. (2014). Global Prostate Cancer Disparities in Black Men. Available at: http://www.cancer.gov/about-nci/organization/cgh/ blog/2014/global-prostate-cancer-disparities-in-black-men (Accessed July 19 2016).

Wootten, A. C., Abbott, J. M., Osborne, D., Austin, D. W., Klein, B., Costello, A. J., \& Murphy, D. G. (2014). The impact of prostate cancer on partners: A qualitative exploration. Psycho-Oncology, 23, 1252-1258.

How to cite this article: Bamidele $\mathrm{O}$, McGarvey $\mathrm{H}$, Lagan BM, et al. Life after prostate cancer: A systematic literature review and thematic synthesis of the post-treatment experiences of Black African and Black Caribbean men. Eur J Cancer Care. 2017;e12784. https://doi.org/10.1111/ecc.12784

\section{APPENDIX A}

\section{Prisma flow chart of search strategy}

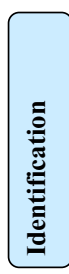

Articles identified from database searches

Medline Ovid (96), PsychInfo (50), CINAHL (81), Embase (186), Cochrane Central (31), Scopus (186), ProQuest Complete (301)
Articles identified from other sources (Grey literature, journal and reference searching, author contact)

$(n=0)$ (irrelevant from titles e.g. relating to breast cancer, oral cancer etc.)

$$
(n=93)
$$

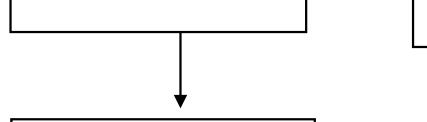

$$
\begin{gathered}
\text { Full-text articles assessed for } \\
\text { eligibility } \\
(n=137)
\end{gathered}
$$

Full-text articles excluded with reasons: Prostate screening $=38$ Intervention studies (mostly related to Caucasian population) $=21$

Prostate cancer epidemiology $=53$

Focused on African American $=15$

Focused on Caucasian men $=6$ $(n=133)$

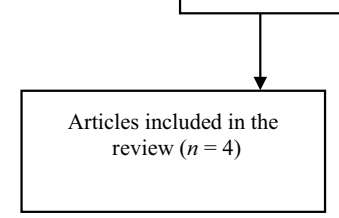




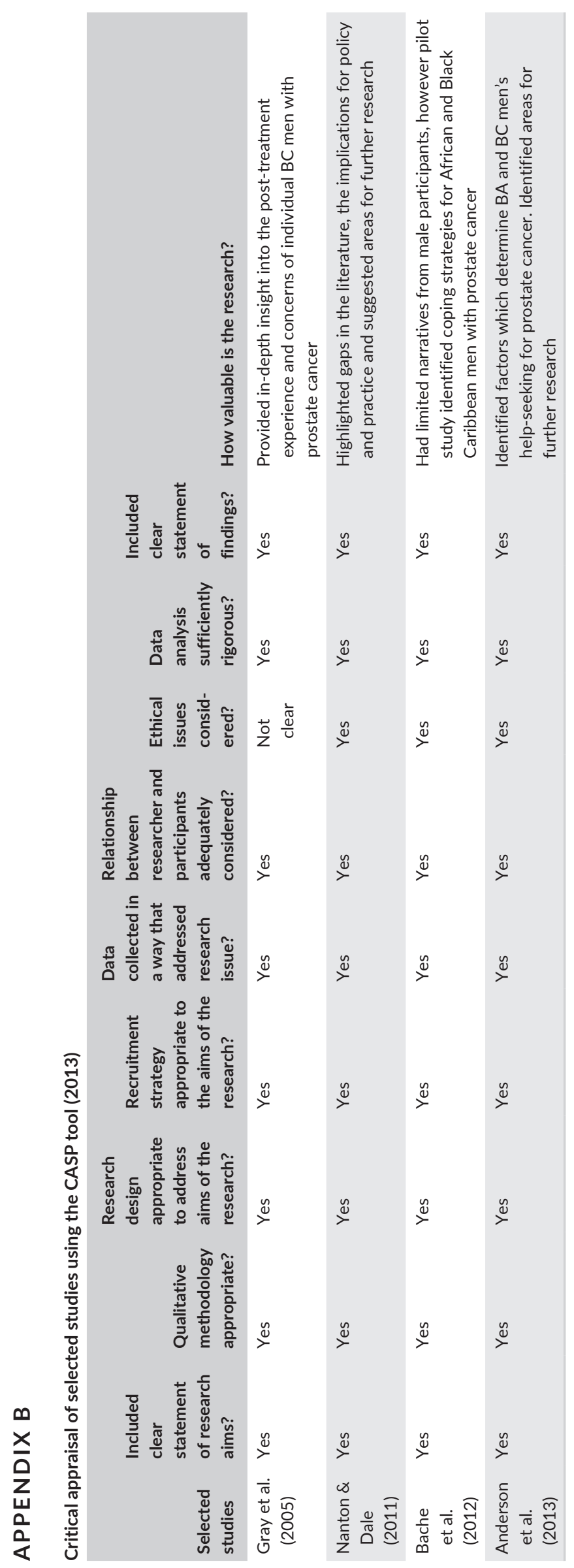

